\title{
Spectral-domain tandem interferometry to measure the group dispersion of optical samples
}

\author{
R. Chlebus, P. Hlubina* , D. Ciprian \\ Department of Physics, Technical University Ostrava, 17. listopadu 15, \\ 70833 Ostrava-Poruba, Czech Republic
}

\begin{abstract}
A spectral-domain technique based on tandem interferometry is used for measuring the group dispersion of optical samples over a wide wavelength range. The technique utilizes a tandem configuration of a Michelson interferometer and an unbalanced Mach-Zehnder interferometer with a sample inserted into its test arm. First, the theoretical background of the technique is presented and then experiments with individual interferometers and their tandem configuration are specified. In all the experiments the spectral signals are recorded to measure the equalization wavelength as a function of the path length difference, or equivalently the group dispersion. We measure the group refractive index as a function of wavelength for a glass sample of known thickness and for a quartz crystal as well.
\end{abstract}

Key words: spectral interferometry, Michelson and Mach-Zehnder interferometer, tandem configuration, group refractive index, dispersion, quartz crystal PACS: 07.60.Ly, 42.25.Hz, 42.25.Lc, 42.81.Gs, 78.20.Fm

* Tel.: +420-597-323-134; fax: +420-597-323-139.

Email address: petr.hlubina@vsb.cz (P. Hlubina). 


\section{Introduction}

The wavelength dependence of the group refractive index, that is, the group dispersion, belongs to one of the fundamental characteristics of both isotropic and anisotropic optical materials. White-light interferometry based on the use of a broadband source in combination with a standard Michelson or MachZehnder interferometer is considered as one of the best tools to measure the group dispersion for different optical materials over a broad spectral range.

White-light interferometry usually utilizes either of two methods, that is, a temporal method or a spectral method, depending on whether interference is observed in the time domain or in the spectral domain, respectively. The timedomain method involves measurement of the time of flight of optical pulses through a sample. A method for measuring the group delay introduced by an optical material consists in placing the sample in one arm of the interferometer and evaluating the temporal shift of the peak of the cross-correlation interferogram. As the central wavelength is varied, the relative group delay of the different frequency components is observed directly [1]. Alternatively, the spectral phase over the full bandwidth of the white-light source can be obtained in a single measurement by a Fourier transform of the cross-correlation interferogram $[2,3]$. The group dispersion of the sample under study can be obtained by simply differentiating the measured spectral phase.

The spectral-domain method is based on the observation of spectrally resolved interference fringes [4-9] in the vicinity of a stationary phase point [4-6] or far from it [7-9] and involves measurement of the phase or period of the spectral fringes. The group dispersion of the sample under study can be obtained by simply differentiating the spectral phase retrieved from a single interferogram. The main limitation of the method is reached for thick or strongly dispersive materials because under such conditions the spectral interference fringes that are far from the stationary phase point become difficult to resolve. Using a lowresolution spectrometer [10], the measurement of the group refractive index dispersion of a given material is still possible in the vicinity of the stationary phase point if one moves it in successive steps to different wavelengths and repeats the measurement. We measured in this way the dispersion of differential group refractive index of pure silica beam splitter present in a Michelson interferometer [10]. The modification of the technique has been used in measurement of the group dispersion of a quartz crystal inserted into a test arm of a Michelson [11] or Mach-Zehnder interferometer [12]. When a birefringent crystal is in tandem with a Michelson interferometer, the dispersion of the group birefringence can only be measured by the technique [13].

In this paper, a spectral-domain technique employing a tandem configuration of two interferometers is used for a direct measurement of the group dispersion 
of optical samples over a wide wavelength range. A Michelson interferometer in tandem with an unbalanced Mach-Zehnder interferometer and a sample inserted into its test arm are employed. The spectral signals are recorded for measuring the equalization wavelength as a function of the overall path length difference or equivalently the group dispersion. In the paper, first, the theoretical background of the technique is presented and then experiments with individual interferometers and their tandem configuration are specified. We measure the group refractive index as a function of wavelength for a glass sample of known thickness and for quartz crystal as well. In the latter case we confirm good agreement between the measured group dispersion and that resulting from the dispersion relation.

\section{Theoretical background}

\subsection{Non-dispersive Michelson interferometer}

Let us consider a configuration of a non-dispersive Michelson interferometer with the adjusted path length difference $\Delta_{\mathrm{M}}$ between beams given by

$$
\Delta_{\mathrm{M}}=2\left(L_{\mathrm{M}}-l_{\mathrm{M}}\right),
$$

where $l_{\mathrm{M}}$ and $L_{\mathrm{M}}$ are the path lengths of the beam in the air in the first and in the second arm of the interferometer, respectively. When a whitelight source is used, a spectral interference signal $S(\lambda)=I(\lambda) / I^{(0)}(\lambda)$ can be recorded by a spectrometer at the output of the interferometer, where $I(\lambda)$ is the spectral intensity with interference and $I^{(0)}(\lambda)$ is the reference spectral intensity (without interference). Let us assume now that the spectrometer is characterized by a Gaussian response function of the width $\Delta \lambda_{\mathrm{R}}$ [14]. The recorded spectral interference signal $S(\lambda)$ is given by [15]

$$
S_{\mathrm{M}}(\lambda)=1+V_{I} \exp \left\{-\left(\pi^{2} / 2\right)\left[\Delta_{\mathrm{M}} \Delta \lambda_{\mathrm{R}} / \lambda^{2}\right]^{2}\right\} \cos \left[(2 \pi / \lambda) \Delta_{\mathrm{M}}\right],
$$

where the visibility term $V_{I}$ is assumed within the resolved spectral interference fringes to be wavelength independent. From Eq. (2), the origin of a spectral modulation of the signal is apparent. If we consider $\Delta_{\mathrm{M}} \geq 0$, the period of spectral modulation $\Lambda(\lambda)$ is given by

$$
\Lambda(\lambda)=\lambda^{2} / \Delta_{\mathrm{M}}
$$

The smaller the path length difference adjusted in the interferometer the larger the period of spectral modulation, which is infinite for the balanced interfe- 
rometer with $L_{\mathrm{M}}=L_{\mathrm{M} 0}=l_{\mathrm{M}}$. From Eq. (2) we can estimate the maximum range of the displacements $\Delta L_{\mathrm{M} \text { max }}$ of mirror 2 of the interferometer, for which the spectral interference fringes are resolvable. When the visibility of the observable spectral interference fringes is assumed to be higher then $0.1 V_{I}$, we obtain

$$
\Delta L_{\mathrm{M} \max }=(2 \sqrt{2 \ln 10} / \pi) \lambda^{2} / \Delta \lambda_{\mathrm{R}} \approx 1.36 \lambda^{2} / \Delta \lambda_{\mathrm{R}}
$$

Assuming a case of a fibre-optic spectrometer with $\Delta \lambda_{\mathrm{R}}=3 \mathrm{~nm}$ [14], the maximum range of the displacements of the interferometer mirror is $\Delta L_{\mathrm{M} \max } \approx$ $90 \mu \mathrm{m}$ for the wavelength range from 450 to $900 \mathrm{~nm}$.

\subsection{Dispersive Michelson interferometer}

Let us consider next that a sample of thickness $t$ and refractive index $n(\lambda)$ is inserted into the first arm of the Michelson interferometer as shown in Fig. 1. The OPD $\Delta_{\mathrm{M}}(\lambda)$ between beams of the dispersive interferometer is given in this case by

$$
\Delta_{\mathrm{M}}(\lambda)=2\left(L_{\mathrm{M}}-l_{\mathrm{M}}\right)-2 t[n(\lambda)-1] .
$$

The recorded spectral interference signal $S(\lambda)$ is given by [15]

$$
S_{\mathrm{M}}(\lambda)=1+V_{I} \exp \left\{-\left(\pi^{2} / 2\right)\left[\Delta_{\mathrm{M}}^{\mathrm{g}}(\lambda) \Delta \lambda_{\mathrm{R}} / \lambda^{2}\right]^{2}\right\} \cos \left[(2 \pi / \lambda) \Delta_{\mathrm{M}}(\lambda)\right],
$$

where $\Delta_{\mathrm{M}}^{\mathrm{g}}(\lambda)$ is the group OPD given by

$$
\Delta_{\mathrm{M}}^{\mathrm{g}}(\lambda)=2\left(L_{\mathrm{M}}-l_{\mathrm{M}}\right)-2 t[N(\lambda)-1],
$$

where $N(\lambda)$ is the group refractive index defined as

$$
N(\lambda)=n(\lambda)-\lambda \frac{\mathrm{d} n(\lambda)}{\mathrm{d} \lambda} .
$$

The corresponding period of spectral modulation is given by Eq. (3) in which the path length difference $\Delta_{\mathrm{M}}$ is replaced by the group OPD $\Delta_{\mathrm{M}}^{\mathrm{g}}(\lambda)$. When the case of thick or strongly dispersive materials is considered, the spectral interference fringes have the largest period in the vicinity of a stationary phase point [4] for which the group OPD is zero at one specific wavelength $\lambda_{0}$, which is referred to as the equalization wavelength [10] and which satisfies the relation

$$
\Delta_{\mathrm{M}}^{\mathrm{g}}\left(\lambda_{0}\right)=2\left(L_{\mathrm{M}}-l_{\mathrm{M}}\right)-2 t\left[N\left(\lambda_{0}\right)-1\right]=0 .
$$


Eq. (9) gives for the mirror position $L_{\mathrm{M}}=L_{\mathrm{M}}\left(\lambda_{0}\right)$ for which the equalization wavelength $\lambda_{0}$ is resolved in the recorded spectral signal the relation

$$
L_{\mathrm{M}}\left(\lambda_{0}\right)=l_{\mathrm{M}}+t\left[N\left(\lambda_{0}\right)-1\right]
$$

If we introduce the mirror displacement $\Delta L_{\mathrm{M}}\left(\lambda_{0}\right)=L_{\mathrm{M}}\left(\lambda_{0}\right)-L_{\mathrm{M} 0}$ as the displacement of mirror 2 of the dispersive interferometer from the mirror position of the balanced non-dispersive Michelson interferometer, we obtain the simple relation

$$
N\left(\lambda_{0}\right)=1+\Delta L_{\mathrm{M}}\left(\lambda_{0}\right) / t
$$

enabling us to measure directly the group refractive index $N\left(\lambda_{0}\right)$ as a function of the equalization wavelength $\lambda_{0}$ for a sample of known thickness $t$.

We can estimate the minimum and maximum thicknesses of the sample whose group dispersion can be measured by the method. If the measurement is restricted to the wavelength range from $\lambda_{0 \min }$ to $\lambda_{0 \max }$ and we assume normal dispersion and no limitation due to the resolving power of the spectrometer, the minimum thickness $t_{\min }$ is given by the minimum displacement $\Delta \mathcal{L}_{\mathrm{M} \text { min }}=\left[\Delta L_{\mathrm{M}}\left(\lambda_{0 \text { min }}\right)-\Delta L_{\mathrm{M}}\left(\lambda_{0 \text { max }}\right)\right]_{\text {min }}$ with which the group dispersion curve is measured

$$
t_{\text {min }}=\Delta \mathcal{L}_{\mathrm{M} \min } /\left[N\left(\lambda_{0 \min }\right)-N\left(\lambda_{0 \max }\right)\right]
$$

Similarly, the maximum thickness $t_{\max }$ is preferably given by the maximum displacement $\Delta L_{\mathrm{M} \max }$ which can be adjusted in the interferometer

$$
t_{\max }=\Delta L_{\mathrm{M} \max } /\left[N\left(\lambda_{0 \min }\right)-1\right] .
$$

Taking into account the material of known dispersion such as fused silica [10] and the spectral range restricted to the wavelengths $\lambda_{0 \min }=450 \mathrm{~nm}$ and $\lambda_{\text {max }}=900 \mathrm{~nm}$, we obtain for $\Delta \mathcal{L}_{\mathrm{M} \text { min }}=100 \mu \mathrm{m}$, which corresponds to ten measurements with a $10 \mu \mathrm{m}$ step, the minimum thickness $t_{\min } \approx 3 \mathrm{~mm}$. Similarly we obtain for $\Delta L_{\mathrm{M} \max }=25 \mathrm{~mm}$, which corresponds to a standard travel of a translation stage, the maximum thickness $t_{\max } \approx 50 \mathrm{~mm}$.

\subsection{Non-dispersive Mach-Zehnder interferometer}

Let us consider a configuration of a non-dispersive Mach-Zehnder interferometer with the path length $l_{\mathrm{MZ}}$ in the air in the first (test) arm and the other 
(reference) arm with the adjustable path length $L_{\mathrm{MZ}}$ in the air, which satisfies the relation $L_{\mathrm{MZ}}>l_{\mathrm{MZ}}$ so that the interferometer is unbalanced. The corresponding path length difference $\Delta_{\mathrm{MZ}}$ between beams is given by

$$
\Delta_{\mathrm{MZ}}=L_{\mathrm{MZ}}-l_{\mathrm{MZ}}
$$

The spectral interference signal $S_{\mathrm{MZ}}(\lambda)$ recorded by a spectrometer of a Gaussian response function is given by

$$
S_{\mathrm{MZ}}(\lambda)=1+V_{I} \exp \left\{-\left(\pi^{2} / 2\right)\left[\Delta_{\mathrm{MZ}} \Delta \lambda_{\mathrm{R}} / \lambda^{2}\right]^{2}\right\} \cos \left[(2 \pi / \lambda) \Delta_{\mathrm{MZ}}\right] .
$$

It results from the previous considerations (see section 2. 1) that due to the large imbalance $\left(\Delta_{\mathrm{MZ}}>>180 \mu \mathrm{m}\right)$ there are available no observable spectral interference fringes. The imbalance can be reduced by inserting a sample into the test arm of the interferometer.

\subsection{Dispersive Mach-Zehnder interferometer}

Let us consider a sample under test of thickness $t$ and refractive index $n(\lambda)$ inserted into the test arm of the unbalanced Mach-Zehnder interferometer as shown in Fig. 2. The OPD $\Delta_{\mathrm{MZ}}(\lambda)$ between the beams in the interferometer is given by

$$
\Delta_{\mathrm{MZ}}(\lambda)=\left(L_{\mathrm{MZ}}-l_{\mathrm{MZ}}\right)-t[n(\lambda)-1] .
$$

Similarly, the group OPD $\Delta_{\mathrm{MZ}}^{\mathrm{g}}(\lambda)$ is given by:

$$
\Delta_{\mathrm{MZ}}^{\mathrm{g}}(\lambda)=\left(L_{\mathrm{MZ}}-l_{\mathrm{MZ}}\right)-t[N(\lambda)-1],
$$

where $N(\lambda)$ is the group refractive index given by Eq. (8). The spectral interference signal $S_{\mathrm{MZ}}(\lambda)$ recorded by a spectrometer of a Gaussian response function is given by

$$
S_{\mathrm{MZ}}(\lambda)=1+V_{I} \exp \left\{-\left(\pi^{2} / 2\right)\left[\Delta_{\mathrm{MZ}}^{\mathrm{g}}(\lambda) \Delta \lambda_{\mathrm{R}} / \lambda^{2}\right]^{2}\right\} \cos \left[(2 \pi / \lambda) \Delta_{\mathrm{MZ}}(\lambda)\right] .
$$

When the case of thick or strongly dispersive materials is considered, the spectral interference fringes recorded in the setup have the largest period at the equalization wavelength $\lambda_{0}$, satisfying the relation $\Delta_{\mathrm{MZ}}^{\mathrm{g}}\left(\lambda_{0}\right)=0$. It gives for the path length $L_{\mathrm{MZ}}=L_{\mathrm{MZ}}\left(\lambda_{0}\right)$ for which the equalization wavelength $\lambda_{0}$ is resolved in the recorded spectrum the relation

$$
L_{\mathrm{MZ}}\left(\lambda_{0}\right)=l+t\left[N\left(\lambda_{0}\right)-1\right] .
$$


If we introduce the path length difference $\Delta L_{\mathrm{MZ}}\left(\lambda_{0}\right)=L_{\mathrm{MZ}}\left(\lambda_{0}\right)-L_{\mathrm{MZ} 0}$, where $L_{\mathrm{MZ}}=L_{\mathrm{MZ0}}=l_{\mathrm{MZ}}$ is the path length for the virtually balanced interferometer (without the sample and with the zero path length difference $\Delta_{\mathrm{MZ}}$ ), we obtain the simple relation

$$
N\left(\lambda_{0}\right)=1+\Delta L_{\mathrm{MZ}}\left(\lambda_{0}\right) / t
$$

enabling us to measure directly the group refractive index $N\left(\lambda_{0}\right)$ as a function of the equalization wavelength $\lambda_{0}$ for a sample of known thickness $t$.

The minimum and maximum thicknesses, $t_{\min }$ and $t_{\max }$, of the sample whose group dispersion can be measured by the method are given by the minimum and maximum path length differences, $\Delta L_{\mathrm{MZ} \text { min }}$ and $\Delta L_{\mathrm{MZ} \max }$, adjustable in the interferometer

$$
t_{\min , \max }=\Delta L_{\mathrm{MZ} \min , \max } /\left[N\left(\lambda_{0 \max , \min }\right)-1\right] .
$$

To determine the group dispersion of the sample under test, we need to known the path length $L_{\mathrm{MZ0}}$ for which is the interferometer balanced and which is not adjustable (see Fig. 2). It can be measured by a method of tandem interferometry based on the fact that the path length difference $\Delta_{\mathrm{MZ}}$ in the Mach-Zehnder interferometer is compensated by the path length difference adjusted in the tandem interferometer such as a Michelson one. Moreover, the group dispersion of samples with thicknesses below $t_{\min }$ can be measured by the same method when the group OPD $\Delta_{\mathrm{MZ}}^{\mathrm{g}}\left(\lambda_{0}\right)$ in the Mach-Zehnder interferometer with the sample is compensated by the path length difference adjusted in the tandem interferometer.

\subsection{Tandem configuration of two non-dispersive interferometers}

Let us consider a tandem configuration of a Michelson interferometer and an unbalanced Mach-Zehnder interferometer that are non-dispersive. The spectral interference signal at the output of the configuration is given by

$$
\begin{aligned}
S_{\mathrm{T}}(\lambda) & =1+V_{I} \exp \left\{-\left(\pi^{2} / 2\right)\left[\Delta_{\mathrm{M}} \Delta \lambda_{\mathrm{R}} / \lambda^{2}\right]^{2}\right\} \cos \left[(2 \pi / \lambda) \Delta_{\mathrm{M}}\right] \\
& +0.5 V_{I} \exp \left\{-\left(\pi^{2} / 2\right)\left[\left(\Delta_{\mathrm{M}}-\Delta_{\mathrm{MZ}}\right) \Delta \lambda_{\mathrm{R}} / \lambda^{2}\right]^{2}\right\} \\
& \times \cos \left[(2 \pi / \lambda)\left(\Delta_{\mathrm{M}}-\Delta_{\mathrm{MZ}}\right)\right] .
\end{aligned}
$$

It results from Eq. (22) that two different types of the spectral interference signals can be resolved. One type is related to the balanced Michelson interferometer configuration and is suitable for determining the position $L_{\mathrm{M} 0}$ and 
the other is related to the Michelson interferometer configuration compensating the path length difference in the unbalanced Mach-Zehnder interferometer and is suitable for determining the position $L_{\mathrm{MZ0}}$.

\subsection{Tandem configuration of two interferometers}

Let us consider a tandem configuration of a Michelson interferometer and an unbalanced Mach-Zehnder interferometer, which is dispersive. The spectral interference signal at the output of the configuration is given by

$$
\begin{aligned}
S_{\mathrm{T}}(\lambda) & =1+V_{I} \exp \left\{-\left(\pi^{2} / 2\right)\left[\Delta_{\mathrm{M}} \Delta \lambda_{\mathrm{R}} / \lambda^{2}\right]^{2}\right\} \cos \left[(2 \pi / \lambda) \Delta_{\mathrm{M}}\right] \\
& +0.5 V_{I} \exp \left\{-\left(\pi^{2} / 2\right)\left[\left(\Delta_{\mathrm{M}}-\Delta_{\mathrm{MZ}}^{\mathrm{g}}(\lambda)\right) \Delta \lambda_{\mathrm{R}} / \lambda^{2}\right]^{2}\right\} \\
& \times \cos \left[(2 \pi / \lambda)\left(\Delta_{\mathrm{M}}-\Delta_{\mathrm{MZ}}(\lambda)\right)\right] .
\end{aligned}
$$

It results from Eq. (23) that once again two different types of the spectral interference signals can be resolved. One type is related to the balanced Michelson interferometer configuration and the other is related to the Michelson interferometer configuration compensating the group OPD $\Delta_{\mathrm{MZ}}^{\mathrm{g}}(\lambda)$ in the MachZehnder interferometer. Using Eqs. (1) and (17), we obtain the relation

$$
N\left(\lambda_{0}\right)=1+\left[\Delta L_{\mathrm{MZ}}\left(\lambda_{0}\right)-2 \Delta L_{\mathrm{M}}\right] / t
$$

enabling us to measure directly the group refractive index $N\left(\lambda_{0}\right)$ as a function of the equalization wavelength $\lambda_{0}$ for a sample of known thickness $t$ when the variable path length difference $\Delta L_{\mathrm{MZ}}\left(\lambda_{0}\right)$ is adjusted in the Mach-Zehnder interferometer.

\section{Experimental set-ups}

The first experimental set-up used in the application of spectral-domain interferometry to measure the dispersion of the group refractive index of a sample is shown in Fig. 1. It consists of a white-light source: a quartz-tungsten-halogen lamp (HL-2000, Ocean Optics, Inc.) with launching optics, optical fibre of cutoff wavelength as short as possible, a collimating lens, a bulk-optic Michelson interferometer, a micropositioner connected to mirror 2 of the interferometer, a microscope objective and micropositioners. The spectral interference fringes are resolved by a fibre-optic spectrometer S2000 (Ocean Optics, Inc.) connected to an A/D converter and a personal computer. The fibre-optic spectrometer S2000 of an asymmetric crossed Czerny-Turner design has spectral 
operation range from 350 to $1000 \mathrm{~nm}$ and includes a 2048-element linear CCDarray. The spectrometer resolution is given by the effective width of the light beam from a core of the read optical fiber and we used the fiber of a $50 \mu \mathrm{m}$ core diameter to which a Gaussian response function with the width $\Delta \lambda_{\mathrm{R}}=3$ nm corresponds [14]. The glass sample, which consists of two polished surfaces, parallel mutually with a sufficient precision, is placed in the first arm of the interferometer in such a way that the collimated beam is incident on the surfaces perpendicularly as shown in Fig. 1.

The second experimental set-up used to measure the group dispersion of a sample under test is shown in Fig. 2. It consists of a bulk-optic Mach-Zehnder interferometer with plate beamsplitters (BSW07, Thorlabs) and the other components that are the same as those in the first set-up. The sample under test is prismatic quartz crystal that consists of polished surfaces with a precision of 15 arcmin. The crystal is placed in the test arm of the interferometer in such a way that the collimated beam, which is parallel to the optic axis of the crystal, is incident on the two surfaces perpendicularly as shown in Fig. 2.

The third experimental set-up used to measure the imbalance of the MachZehnder interferometer and the group dispersion of optical samples corresponds to a tandem configuration of the set-ups shown in Figs. 1 and 2. The first set-up is without the sample and the second one is either non-dispersive or includes the glass sample or the quartz crystal.

\section{Experimental results and discussion}

Prior to the measurement of the group refractive index dispersion of the glass sample in the first set-up, the position $L_{\mathrm{M} 0}$ of mirror 2 for which the nondispersive Michelson interferometer is balanced was determined. We utilized the fact that for this position the spectral interference fringes have the largest period of modulation. Then we inserted the glass sample having the thickness $t=(15850 \pm 10) \mu \mathrm{m}$ into the first arm of the interferometer (see Fig. 1) and displaced mirror 2 to such a position to resolve spectral interference fringes. Fig. 3 shows an example of the spectral signal recorded for the mirror displacement $\Delta L_{\mathrm{M}}=8461 \mu \mathrm{m}$ and clearly demonstrates the effect of the limiting resolving power of the spectrometer on the visibility of the spectral interference fringes identified only over a narrow spectral range in the vicinity of the equalization wavelengths $\lambda_{0}=661.94 \mathrm{~nm}$. We measured in this way the dependence of the equalization wavelength $\lambda_{0}$ on the adjusted displacement $\Delta L_{\mathrm{M}}$ of mirror 2 . We displaced mirror 2 manually by using the micropositioner with a constant step of $10 \mu \mathrm{m}$ and performed recording of the corresponding spectral signals. The recorded spectral signals have revealed that the equalization wavelengths 
$\lambda_{0}$ can be resolved in the spectral range from 455 to $950 \mathrm{~nm}$ and that the corresponding displacement $\Delta L_{\mathrm{M}}$ varies from 8951 to $8271 \mu \mathrm{m}$.

Using the measured dependence of the displacement $\Delta L_{\mathrm{M}}\left(\lambda_{0}\right)$ on the equalization wavelength $\lambda_{0}$ and Eq. (11), we evaluated directly for the known thickness $t$ the group refractive index $N\left(\lambda_{0}\right)$ of the glass sample as a function of the equalization wavelength $\lambda_{0}$. The function is represented in Fig. 4 by the crosses and it is shown together with a polynomial fit. We can estimate a precision of the group refractive index measurement. If the equalization wavelength is determined with an error of $\delta\left(\lambda_{0}\right)$, the displacement $\Delta L_{\mathrm{M}}$ adjusted in the interferometer is known with a precision of $\delta\left(\Delta L_{\mathrm{M}}\right)$ and the thickness of the glass sample is known with a precision of $\delta(t)$, the group refractive index $N$ is obtained with a precision given by the following formula

$$
\delta(N)=\sqrt{\left[\frac{\mathrm{d} N\left(\lambda_{0}\right)}{\mathrm{d} \lambda_{0}} \delta\left(\lambda_{0}\right)\right]^{2}+\left[\frac{\delta\left(\Delta L_{\mathrm{M}}\right)}{t}\right]^{2}+\left[\Delta L_{\mathrm{M}} \frac{\delta(t)}{t^{2}}\right]^{2}} .
$$

In our case, the error $\delta\left(\lambda_{0}\right)$ is $0.32 \mathrm{~nm}$ and corresponds to the wavelength difference of adjacent pixels of the spectrometer linear CCD-array detector, the precision $\delta\left(\Delta L_{\mathrm{M}}\right)$ is $1 \mu \mathrm{m}$ and the precision $\delta(t)$ is $10 \mu \mathrm{m}$ so that the precision $\delta(N)$ in obtaining the group refractive index is $4 \times 10^{-4}$. Higher measurement precision can be achieved using a glass sample, the thickness of which is known with higher precision and/or is larger. However, the maximum thickness of the glass sample is limited by resolving power of the spectrometer.

Prior to the measurement of the group refractive index dispersion of the quartz crystal in the second set-up, the virtual (not adjustable) path length $L_{\mathrm{MZ0}}$ corresponding to the balanced Mach-Zehnder interferometer was determined. We used a tandem configuration of the Michelson interferometer and the unbalanced Mach-Zehnder interferometer with the adjusted path length $L_{\mathrm{MZ}}$. We used the fact that the spectral interference fringes are observable for the zero path length difference in the Michelson interferometer and for such a path length difference which is the same as the one adjusted in the unbalanced Mach-Zehnder interferometer. The adjusted path length difference $\Delta L_{\mathrm{MZ}}=L_{\mathrm{MZ}}-L_{\mathrm{MZ0}}$ is determined in this way with a precision better then $1 \mu \mathrm{m}$. Then the group dispersion of a quartz crystal having the thickness $t=(40890 \pm 10) \mu \mathrm{m}$ was measured in the set-up shown in Fig. 2. The main advantage of the set-up is in fibre connection of a light source (that can be varied) with the interferometer. Using a laser diode instead of the halogen lamp, we checked precise placement of the crystal sample in the test arm by observing the interference fringes.

The group dispersion of the quartz crystal was measured by adjusting such a path length to resolve spectral interference fringes. Fig. 5 shows an example of the spectral signal recorded for the path length difference $\Delta L_{\mathrm{MZ}}=23000 \mu \mathrm{m}$ 
with the spectral interference fringes identified only in the vicinity of the equalization wavelength $\lambda_{0}=648.23 \mathrm{~nm}$. We measured in this way the dependence of the adjusted path length difference on the equalization wavelength. We displaced the stage with mirrors 3 and 4 manually by using the micropositioner with a constant step of $10 \mu \mathrm{m}$ and performed recording of the corresponding spectral signals. The recorded spectral signals revealed that the equalization wavelength $\lambda_{0}$ can be resolved in the spectral range from 490 to $899 \mathrm{~nm}$ and that the path length difference $\Delta L_{\mathrm{MZ}}$ varies from 23820 to $22540 \mu \mathrm{m}$. Using the measured dependence of the displacement $\Delta L_{\mathrm{MZ}}\left(\lambda_{0}\right)$ on the equalization wavelength $\lambda_{0}$ and Eq. (20), we evaluated directly for the known thickness $t$ the group refractive index $N\left(\lambda_{0}\right)$ of the quartz crystal as a function of the equalization wavelength $\lambda_{0}$. The function is represented in Fig. 6 by the crosses and it is shown together with the theoretical function resulting from the dispersion relation for the extraordinary polarization in the birefringent quartz $[11,16]$. The group refractive index $N$ is obtained with a precision of $1 \times 10^{-4}$ resulting from Eq. (25).

Then we measured the group dispersion of the glass sample inserted into the test arm of the Mach-Zehnder interferometer. Because the sample thickness is smaller than the limiting one given by Eq. (21), the third set-up with a tandem configuration of the Michelson interferometer and the unbalanced MachZehnder interferometer was used. First, the position of mirror 2 in the Michelson interferometer was adjusted to resolve the spectral interference fringes. Then the path length difference $\Delta L_{\mathrm{MZ}}$ in the Mach-Zehnder interferometer was varied to resolve the other spectral interference fringes and the dependence of the equalization wavelength $\lambda_{0}$ on the adjusted overall path length difference $\Delta L=\Delta L_{\mathrm{MZ}}-2 \Delta L_{\mathrm{M}}$ was determined. As an example, Fig. 7 shows the spectral signal recorded for the path length difference $\Delta L=8537 \mu \mathrm{m}$ with the spectral interference fringes identified only in the vicinity of the equalization wavelength $\lambda_{0}=608.01 \mathrm{~nm}$. Using Eq. (24) and the known sample thickness $t$ we evaluated directly the group refractive index $N\left(\lambda_{0}\right)$ of the glass sample as a function of the equalization wavelength $\lambda_{0}$. The function is represented in Fig. 8 by the crosses and it is shown together with the function obtained by the measurement with the first set-up. Fig. 8 demonstrates good compatibility of both measurement methods.

Finally, we used the third set-up to measure the group dispersion of the quartz crystal having the thickness $t=(20950 \pm 10) \mu \mathrm{m}$, which is once again smaller than the limiting one given by Eq. (21). The optic axis of the crystal, which was inserted in the test arm of the Mach-Zehnder interferometer, was perpendicular to the beams and mirror 2 in the Michelson interferometer was displaced to such a position to resolve spectral interference fringes. Fig. 9 shows an example of the spectral signal recorded for the overall path length difference $\Delta L=11998 \mu \mathrm{m}$. In this spectral signal are resolved two types of interference fringes. First type of interference fringes is located in a range of 
shorter wavelengths and corresponds to the ordinary polarization and the second type of the interference fringes is located in a range of longer wavelengths and corresponds to the extraordinary polarization. The spectral interference fringes are identified only over narrow spectral ranges in the vicinities of the equalization wavelengths $\lambda_{o}=552.31 \mathrm{~nm}$ and $\lambda_{e}=646.59 \mathrm{~nm}$. We measured in this way the dependence of the equalization wavelengths corresponding to the ordinary or extraordinary polarizations on the adjusted overall path length difference $\Delta L$. Using Eq. (24) and the measured dependences of the path length difference $\Delta L_{o}\left(\lambda_{o}\right)$ on the equalization wavelength $\lambda_{o}$ and the path length difference $\Delta L_{e}\left(\lambda_{e}\right)$ on the equalization wavelength $\lambda_{e}$, we evaluated directly for the known thickness $t$ the group refractive indices $N_{o}\left(\lambda_{o}\right)$ and $N_{e}\left(\lambda_{e}\right)$ of the quartz crystal as a function of the equalization wavelengths $\lambda_{o}$ and $\lambda_{e}$. These functions are represented in Fig. 10 by the crosses and they are shown together with the theoretical functions given by the dispersion relation for the extraordinary and ordinary polarizations in the birefringent quartz $[11,16]$. The precision of the group refractive indices measurement is $3 \times 10^{-4}$.

\section{Conclusions}

We used a spectral-domain technique based on tandem interferometry for measuring the group dispersion of optical samples over a wide wavelength range. The technique utilizes a Michelson interferometer in tandem with an unbalanced Mach-Zehnder interferometer and a sample inserted into its test arm. First, we presented the theoretical background of the technique and then we specified experiments with individual interferometers and their tandem configuration as well. In all the experiments the spectral signals were recorded to measure the equalization wavelength as a function of the path length difference, or equivalently the group dispersion. We measured the group refractive index as a function of wavelength for a glass sample of known thickness. We also measured the group dispersion for a quartz crystal and confirmed good agreement between the measured group dispersion and that resulting from the dispersion relation. The technique is applicable to any dispersive component with arbitrarily high dispersion or thickness, provided that the spectral interference fringes are resolved.

Acknowledgements The research was partially supported by the Grant Agency of the Czech Republic (projects 102/06/0284, 202/06/0531), by the grant MSM6198910016 and by internal grant of TU Ostrava (IGS HGF VŠBTUO). 


\section{References}

[1] Knox WH, Pearson NM, Li KD, Hirlimann CA. Interferometric measurements of femtosecond group delay in optical components. Opt Lett 1988;13:574-6.

[2] Diddams S, Diels JC. Dispersion measurements with white-light interferometry. J Opt Soc B 1995;13:1120-8.

[3] Galli M, Marabelli F, Gizzetti G. Direct measurement of refractive-index dispersion of transparent media by white-light interferometry. Appl Opt $2003 ; 42: 3910-391$.

[4] Sáinz J, Jourdain P, Escalona R, Calatroni J. Real time interferometric measurements of dispersion curves. Opt Commun 1994;110:381-90.

[5] Kumar VN, Rao DN. Using interference in the frequency domain for precise determination of the thickness and refractive indices of normal dispersive materials. J Opt Soc B 1995;12:1559-63.

[6] Delbarre H, Przygodzki C, Tassou M, Boucher D. High-precision index measurement in anisotropic crystals using white-light spectral interferometry. Appl Phys B 2000;70:45-51.

[7] Liang Y, Grover CH. Modified white-light Mach-Zehnder interferometer for direct group-delay measurements. Appl Opt 1998;37:4105-11.

[8] Lee JY, Kim DY. Versatile chromatic dispersion measurement of a single mode fiber using spectral white light interferometry. Opt Express 2006;14:11608-15.

[9] Reolon D, Jacquot M, Verrier I, Brun G, Veillas C. High resolution group refractive index measurement by broadband supercontinuum interferometry and wavelet-transform analysis. Opt Express 2006;14:12744-50.

[10] Hlubina P. White-light spectral interferometry with the uncompensated Michelson interferometer and the group refractive index dispersion in fused silica. Opt Commun 2001;193:1-7.

[11] Hlubina P, Ciprian D, Knyblová L. Direct measurement of dispersion of the group refractive indices of quartz crystal by white-light spectral interferometry. Opt Commun 2007;269:8-13.

[12] Chlebus R, Hlubina P, Ciprian D. Direct measurement of group dispersion of optical components using white-light spectral interferometry. Opto-Electronics Review 2007;15:144-8.

[13] Hlubina P, Urbanczyk W. Dispersion of the group birefringence of a calcite crystal measured by white-light spectral interferometry. Meas Sci Technol $2005 ; 16: 1267-71$.

[14] Hlubina P, Gurov I, Chugunov V. White-light spectral interferometric technique to measure the wavelength dependence of the spectral bandpass of a fibre-optic spectrometer. J Mod Opt 2003;50:2067-74. 
[15] Hlubina P. Dispersive spectral-domain two-beam interference analysed by a fibre-optic spectrometer. J Mod Opt 2004;51:537-47.

[16] Ghosh G. Dispersion-equation coefficients for the refractive index and birefringence of calcite and quartz crystal. Opt Commun 1999;163:95-102. 


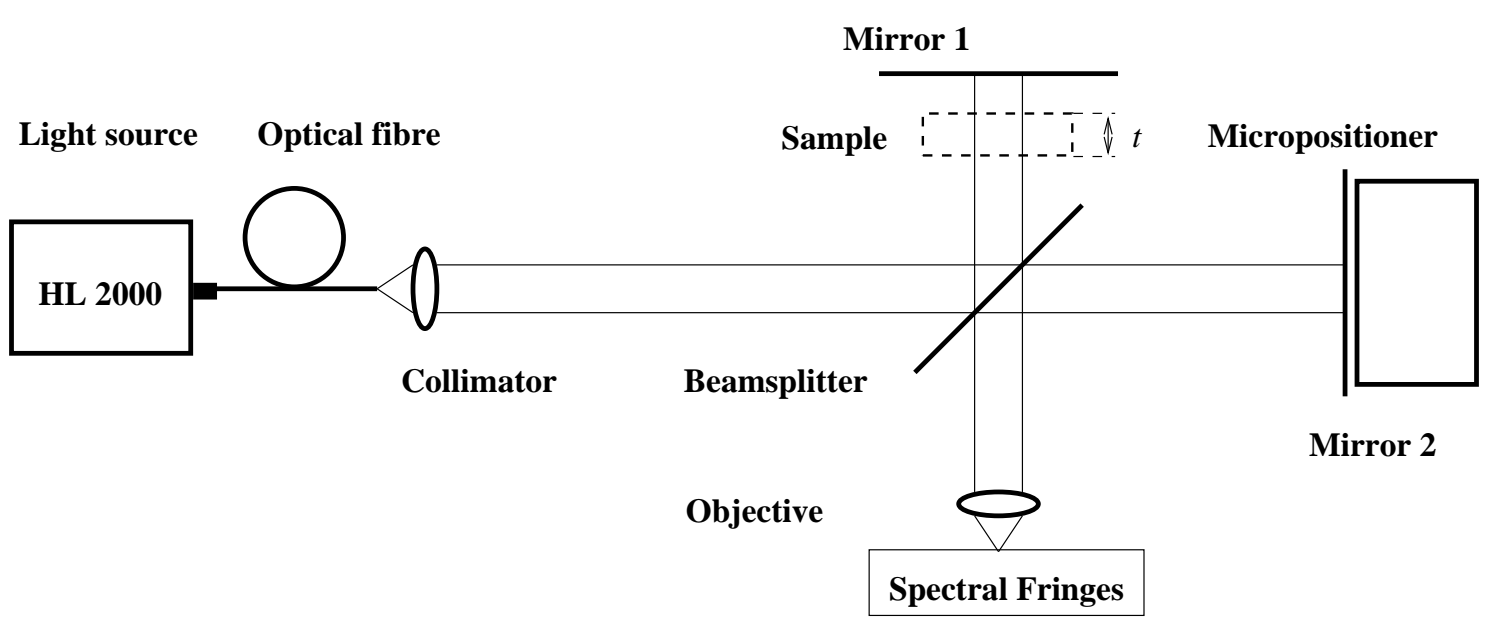

Fig. 1. The first experimental set-up with a Michelson interferometer.

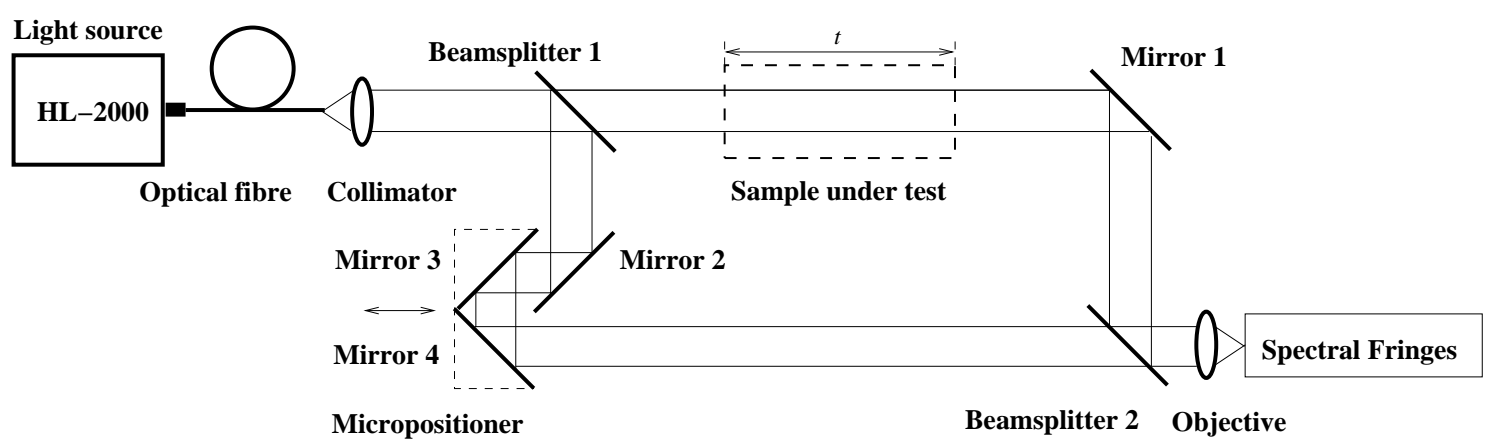

Fig. 2. The second experimental set-up with an unbalanced Mach-Zehnder interferometer. 


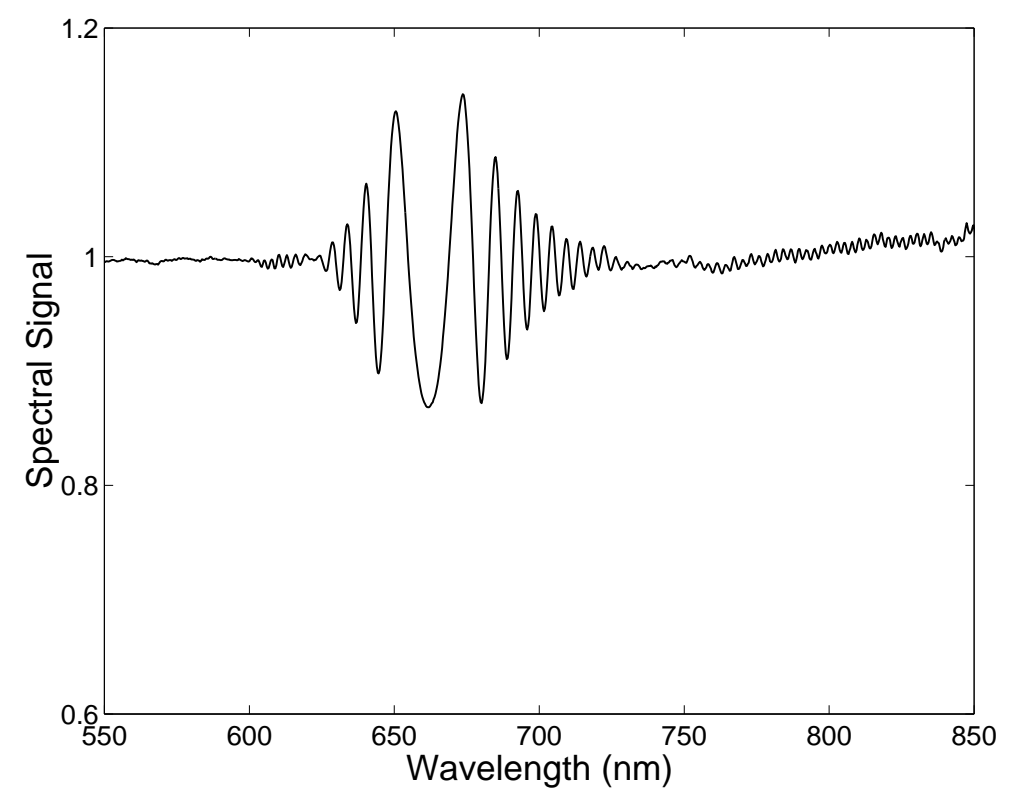

Fig. 3. Example of the spectral signal recorded in the first set-up for a glass sample.

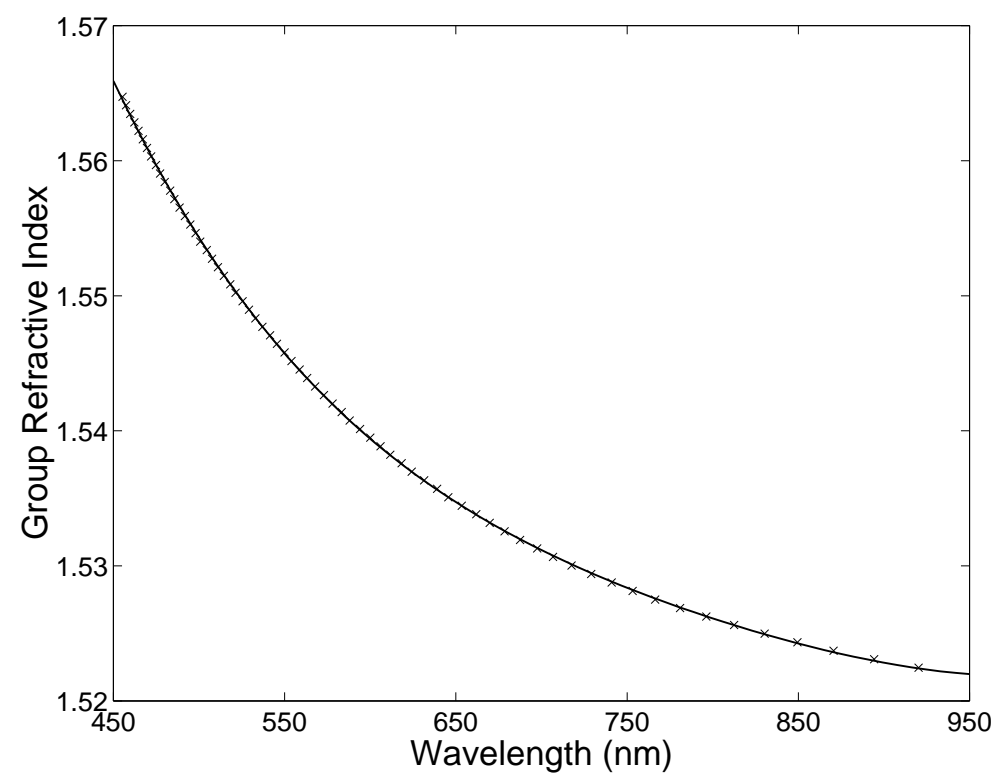

Fig. 4. The group refractive index of the glass sample as a function of the wavelength (solid line is a polynomial fit). 


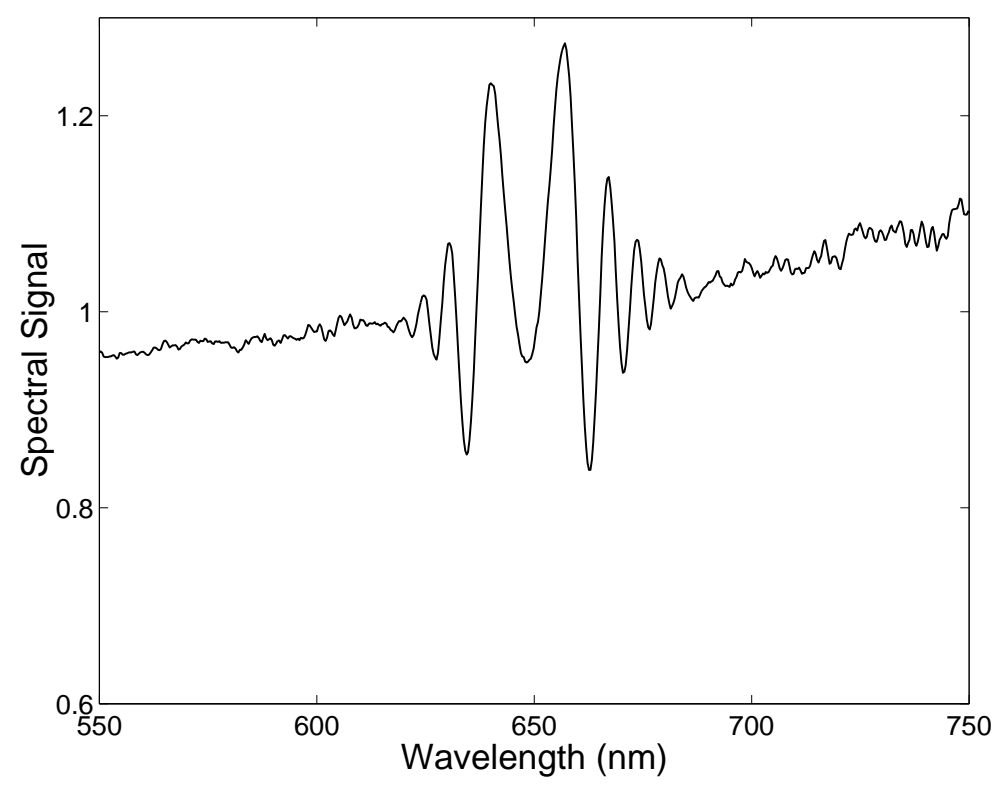

Fig. 5. Example of the spectral signal recorded in the second set-up for the quartz crystal.

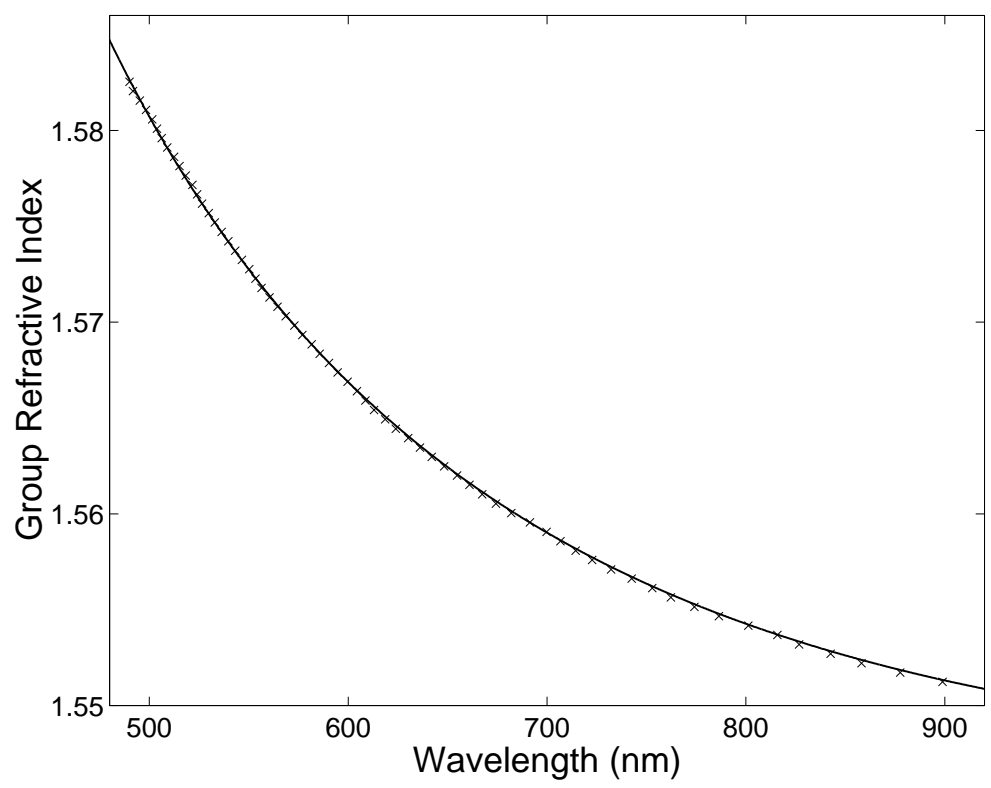

Fig. 6. The group refractive index of the quartz crystal as a function of the wavelength (solid line corresponds to theory). 


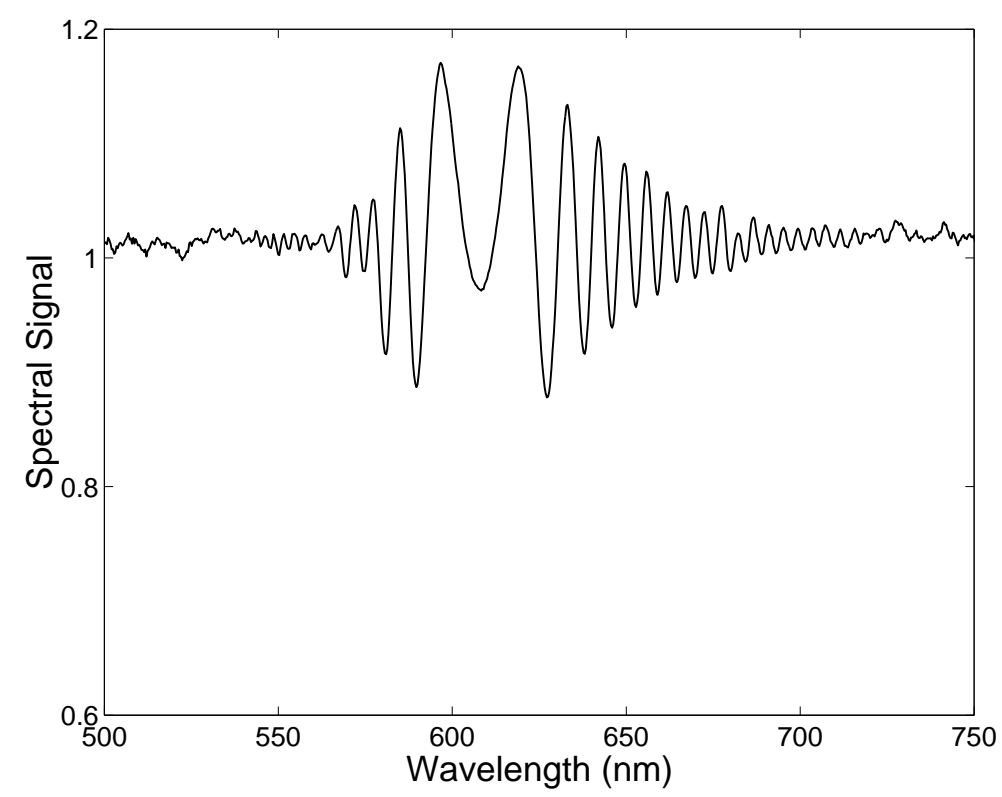

Fig. 7. Example of the spectral signal recorded in a tandem configuration of the first and second set-up for the glass sample.

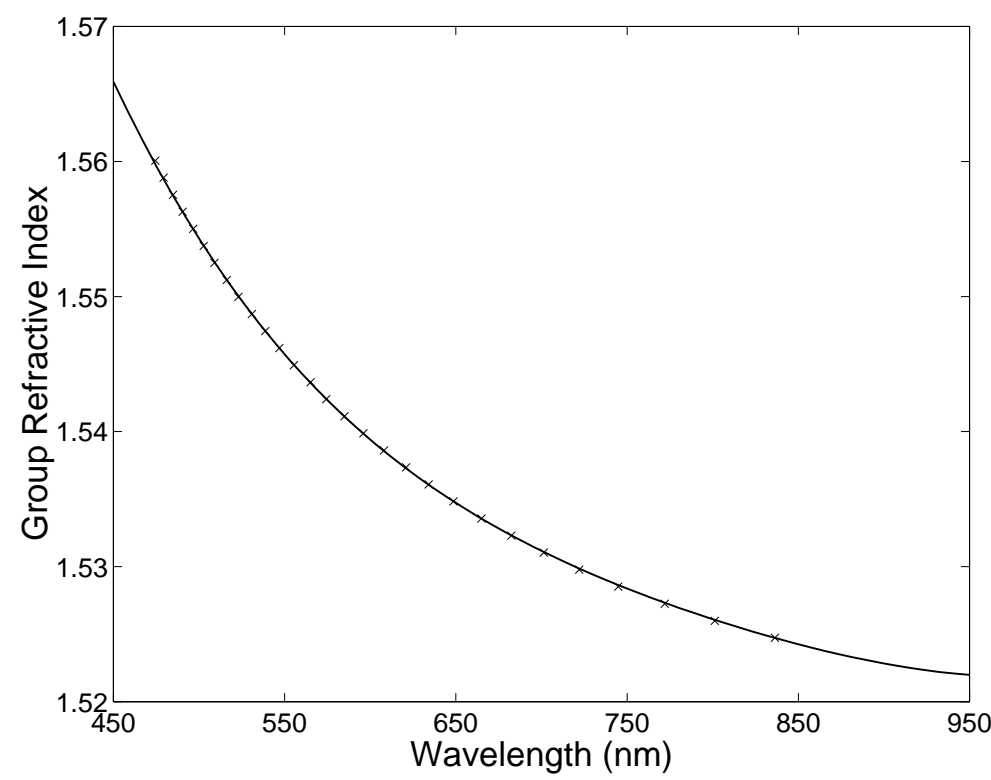

Fig. 8. The group refractive index of the glass sample as a function of the wavelength (solid line is polynomial fit from Fig. 4). 


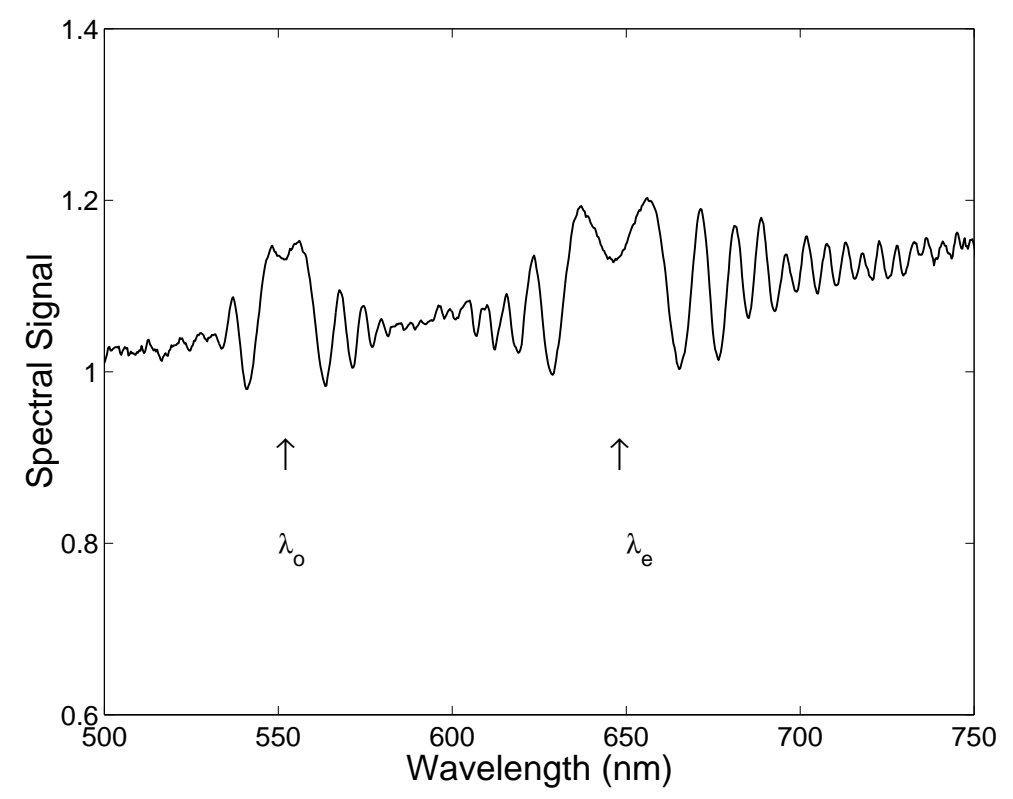

Fig. 9. Example of the spectral signal recorded in a tandem configuration of the first and second set-up for the quartz crystal.

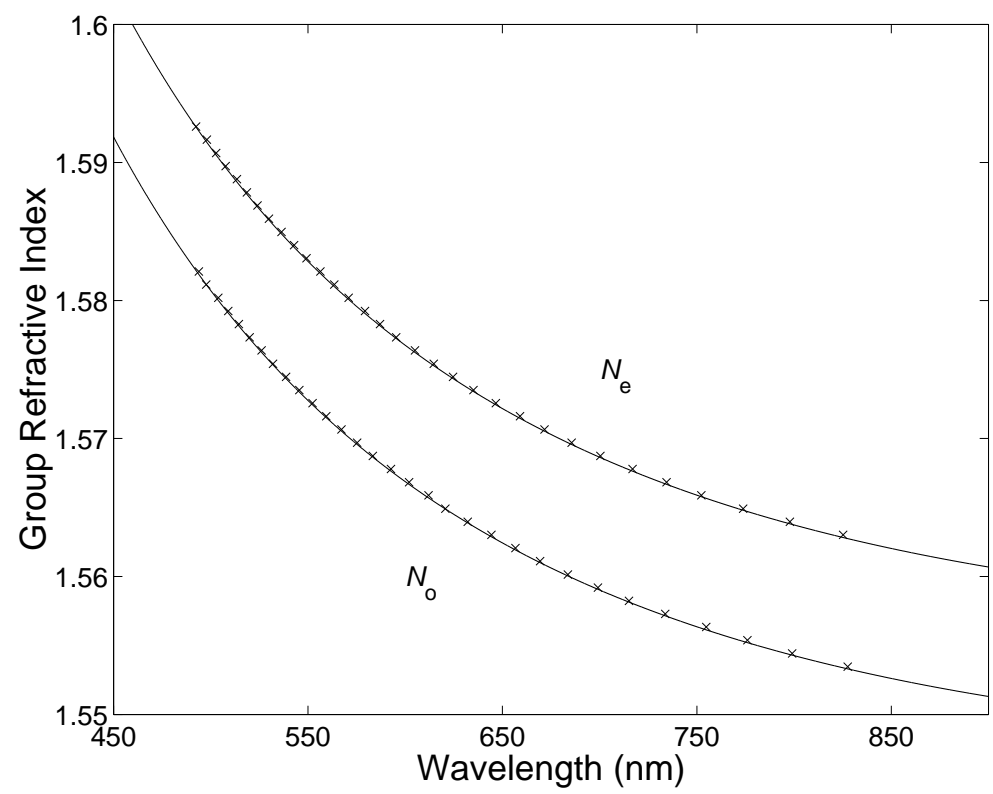

Fig. 10. The group refractive index of the quartz crystal as a function of the wavelength (solid lines correspond to theory). 